\title{
A Multicenter Comparative Study of Cefepime Versus Broad-Spectrum Antibacterial Therapy in Moderate and Severe Bacterial Infections
}

\author{
Roberto Badaró, Fernando Molinar, Carlos Seas, \\ Daniel Stamboulian, João Mendonça, João Massud, \\ Luiz Olympio Nascimento, on behalf of the Latin \\ American Antibiotic Research Group (LAARG)
}

\author{
University Hospital Professor Edgard Santos, Salvador, \\ BA, Servidor Público Hospital, Bristol Myers Squibb \\ Brazil, São Paulo, SP, Brazil; Medical Center "La Raza," \\ Institute of Social Security, Mexico; Nacional Hospital \\ Cayetano, Institute of Tropical Medecine "Alexander von \\ Humboldt," Lima, Peru; Foundation for the Study of \\ Infectious Diseases (FUNCEI), B. Aires, Argentina
}

The safety and efficacy of cefepime empiric monotherapy compared with standard broad-spectrum combination therapy for hospitalized adult patients with moderate to severe community-acquired bacterial infections were evaluated. In an open-label, multicenter study, 317 patients with an Acute Physiology and Chronic Health Evaluation (APACHE II) score ranging from $>5$ to $=19$ were enrolled with documented pneumonia ( $n=196)$, urinary tract infection $(n=65)$, intra-abdominal infection $(n=38)$, or sepsis $(n=18)$. Patients were randomly assigned 1:1 to receive cefepime 1 to $2 \mathrm{~g} \mathrm{IV} \mathrm{twice} \mathrm{daily} \mathrm{or} \mathrm{three} \mathrm{times} \mathrm{a} \mathrm{day} \mathrm{or} \mathrm{IV}$ ampicillin, cephalothin, or ceftriaxone \pm aminoglycoside therapy for 3 to 21 days. For both treatment groups, metronidazole, vancomycin, or macrolide therapy was added as deemed necessary. The primary efficacy variable was clinical response at the end of therapy. Two hundred ninety-six $(93 \%)$ patients met evaluation criteria and were included in the efficacy analysis. Diagnoses included the following: 180 pneumonias ( 90 cefepime, 90 comparator), 62 urinary tract infections ( 29 cefepime, 33 comparator), 37 intra-abdominal infections (19 cefepime, 18 comparator), and 17 sepses ( 8 cefepime, 9 comparator). At the end of therapy, overall clinical success rates were $131 / 146(90 \%)$ for patients treated with cefepime vs $125 / 150(83 \%)$ for those treated with comparator ( $95 \%$ confidence interval $[C I]:-2.6 \%$ to $16.3 \%)$. The clinical success rate for patients with community-acquired pneumonia, the most frequent infection, was $86 \%$ for both treatment groups. Among the patients clinically evaluated, 162 pathogens were isolated and identified before therapy. The most commonly isolated pathogens were Escherichia coli $(\mathrm{n}=49)$, Streptococcus pneumoniae $(\mathrm{n}=29)$, Haemophilus influenzae $(\mathrm{n}=14)$, and Staphylococcus aureus $(n=11)$. Bacteriologic eradication/presumed eradication was $97 \%$ for cefepime vs $94 \%$ for comparatortreated patients. Drug-related adverse events were reported in $16 \%$ of cefepime patients and $19 \%$ of comparator patients. In conclusion, cefepime had higher cure rates compared with broad-spectrum combination therapy as an initial empiric treatment for hospitalized patients with moderate to severe community-acquired infections, including urinary tract infections, intra-abdominal infections, and sepsis. Key Words: Cefepime, ampicillin, caphalotin, ceftriaxone, aminoglycoside, urinary tract infections, intra-abdominal infections, sepsis.

Received on 15 July 2002; revised 31 August 2002.

Address for correspondence: Dr. Roberto Badaró, $\mathrm{MD}, \mathrm{PhD}$ Hosp. Prof. Edgard Santos - $6^{\circ}$ andar -Infectologia. Rua João das Botas, s/n Canela,40110-160. Salvador, Bahia, Brazil. Phone: 5571 235-4901. Fax: 5571 247-2756.

This work was supported by a research grant from BristolMyers Squibb Company, Princeton, New Jersey, and Fundação Bahiana de Infectologia, Salvador, Bahia, Brazil.

The Brazilian Journal of Infectious Diseases 2002;6(5):206-218 (C) 2002 by The Brazilian Journal of Infectious Diseases and Contexto Publishing. All rights reserved.

$1413-8670$
The initial selection of an empiric antimicrobial regimen for the treatment of hospitalized patients with serious community-acquired infections requires the use of broadspectrum antibiotics. It has been common practice to treat presumed bacterial infections (e.g. pneumonia, urinary tract infection, sepsis) with combination antibiotic therapy, such as a $\beta$-lactam plus an aminoglycoside, in order to cover likely Gram-positive and Gram-negative organisms. In addition, combination therapy is prescribed to provide synergy against difficult-to-treat pathogens or ones that are likely to emerge resistant (e.g. Staphylococcus aureus, 
Enterococcus spp, Pseudomonas aeruginosa) [1]. The past decade has witnessed the development of agents with broad spectrums of in vitro activity and has permitted the option ofmonotherapy in select patients [2-4]. The potential advantages of monotherapy include decreased risk for toxicity and drug interactions, reduced drug expenditures, and other pharmacoeconomic benefits [5-7].

Before an initial empiric antimicrobial regimen for the treatment of serious community-acquired infections is prescribed, local susceptibility patterns must be considered [1]. In Latin America, antimicrobial resistance has escalated against commonly isolated community-acquired pathogens from the respiratory tract $[8,9]$, urinary tract [10], and blood [11].

Cefepime is a potent, broad-spectrum, fourthgeneration cephalosporin with enhanced activity against many Gram-positive and Gram-negative aerobic bacteria, including multiply resistant strains of Enterobacteriaceae [12-16]. In addition, cefepime has excellent in vitro activity against methicillin-susceptible $S$. aureus and $P$. aeruginosa. Cefepime's broad spectrum of in vitro activity is attributable to its low affinity for most $\beta$ lactamases (especially Bush group 1) [17], its high affinity for essential penicillin-binding proteins, and its zwitterionic structure [12-16]. Cefepime also appears to have a low propensity toward the development of resistance.

The primary purpose of this trial was to evaluate the efficacy and safety of cefepime monotherapy compared with standard broad-spectrum antimicrobial combination regimens as initial empiric treatment of hospitalized adults with moderate to severe infections; these infections include community-acquired pneumonia (CAP), urinary tract infection (UTI), intra-abdominal infection, and sepsis. This study was conducted in the 4 Latin American countries of Argentina, Brazil, Mexico, and Peru.

\section{Material and Methods}

Study design and initial antimicrobial therapy

\section{Inclusion criteria}

This was a prospective, open-label, randomized, multicenter study conducted between June 1999 and
March 2000 at 34 centers in Mexico, Brazil, Argentina, and Peru. After satisfying enrollment criteria and providing informed consent, patients were randomly assigned 1:1 to receive cefepime 1 or $2 \mathrm{~g}$ IV twice daily or three times a day (moderate or severe infections, respectively) or a standard antimicrobial combination, according to the investigator's discretion or the center's usual regimen. Standard antimicrobial combinations included the following: 1) ampicillin 2 to $3 \mathrm{~g}$ IV four times daily \pm gentamicin $80 \mathrm{mg}$ IV two times daily or three times a day or amikacin $500 \mathrm{mg}$ IV three times daily; 2) cephalothin 2 to $3 \mathrm{~g}$ IV four times daily \pm gentamicin $80 \mathrm{mg}$ IV twice daily or three times a day or amikacin 500mg IV three times daily; or 3 ) ceftriaxone 1 to $2 \mathrm{~g}$ IV twice daily \pm gentamicin $80 \mathrm{mg}$ IV twice daily or three times a day or amikacin $500 \mathrm{mg}$ IV three times daily. For both treatment groups, metronidazole was added if anaerobes were suspected, vancomycin was given ifmethicillin-resistant $S$. aureus was suspected or documented, and macrolides were added for patients with CAP, if deemed appropriate (Figure 1). In the event that a pretherapy culture revealed at least 1 pathogen resistant to study drug, the patient was allowed to continue to receive the assigned antimicrobial, unless the investigator deemed that an alternative antimicrobial was necessary.

Eligible patients were men and women who were at least 18 years of age with a clinical diagnosis of moderate to severe CAP, UTI, intra-abdominal infection, or sepsis. All participants required hospitalization for their infection for less than 3 days prior to study entry. Each patient had an Acute Physiology and Chronic Health Evaluation (APACHE) II [18] score ranging from more than 5 to 19 or less.

Enrollment criteria for patients entering the pneumonia arm of the study included a new infiltrate on chest X-ray plus at least 2 clinical signs/symptoms, such as fever $\left(>38^{\circ} \mathrm{C}\right.$ or $\left.>100.4^{\circ} \mathrm{F}\right)$; leukocytosis $\left(>10,000\right.$ white blood cells $[\mathrm{WBCs}] / \mathrm{mm}^{3}$ or $>15 \%$ bands); cough; purulent sputum ( $>25$ polymorphonuclear leukocytes [PMNs] and $<10$ squamous epithelial cells per low power field); chest pain; auscultatory findings (e.g. rales or egophony); chills; headache; or malaise. 
The diagnosis of UTI required the isolation of more than $10 \mathrm{WBC}$ /high power field (HPF) obtained from centrifuged urine, collected by clean-catch technique or by catheterization, and at least 2 of the following: fever $\left(>38^{\circ} \mathrm{C}\right.$ or $\left.>100.4^{\circ} \mathrm{F}\right)$; leukocytosis $(>10,000$ $\mathrm{WBCs} / \mathrm{mm}^{3}$ or $>15 \%$ bands); or upper tract symptoms (flank or back pain or costovertebral angle tenderness).

Individuals entering the bacterial sepsis arm of the study had to have clinical evidence of infection, including fever $\left(>38^{\circ} \mathrm{C}\right.$ or $\left.>100.4^{\circ} \mathrm{F}\right)$; tachycardia $(=90$ beats per minute [bpm]); leukocytosis $\left(>10,000 \mathrm{WBCs} / \mathrm{mm}^{3}\right.$ or $>15 \%$ bands); respiratory frequency of 20 or more; increased arterial carbon dioxide tension $\left(\mathrm{PaCO}_{2}\right)$ of less than $32 \mathrm{~mm} / \mathrm{Hg}$; and evidence or suspicion of infection in another site. In addition, each patient must have had evidence of at least 1 of the following: hypotension (reduced systolic and diastolic blood pressures of $20 \mathrm{~mm} / \mathrm{Hg}$ and $10 \mathrm{~mm} / \mathrm{Hg}$, respectively, below the patient's baseline for at least 8 hours in the absence of an obvious cause other than sepsis); oliguria ( $<15 \mathrm{~mL} / \mathrm{h}$ urine during 4 hours); or hyperventilation (respiratory rate $>25 \mathrm{bpm}$, or an increase of $15 \mathrm{bpm}$ above the patient's baseline over 4 hours, in the absence of an obvious cause other than sepsis).

The diagnosis of intra-abdominal infection required clinical evidence, such as abdominal tenderness or hypoactive bowel sounds, and at least 2 of the following: fever $\left(>38^{\circ} \mathrm{C}\right.$ or $\left.>100.4^{\circ} \mathrm{F}\right)$; leukocytosis ( $>10,000 \mathrm{WBCs} / \mathrm{mm}^{3}$ or $>15 \%$ bands); radiographic, computed tomographic, or ultrasonographic findings suggestive of perforation or abscess; or documentation of a perforation or abscess at the time of surgery.

\section{Exclusion criteria}

Patients were excluded from the study if they had any of the following characteristics or conditions: APACHE II scores of 5 or lower or higher than 19; pregnancy and/or lactation; limited life expectancy (i.e. $<3$ days, or patients on "do not resuscitate" status); clinically significant hepatic disease (i.e. alanine aminotransferase and/or aspartate aminotransferase and/or total bilirubin $\geq 5$ times the upper limit of normal); chronic renal insufficiency (e.g. serum creatinine $=3.0 \mathrm{mg} / \mathrm{dL}$ or requiring renal dialysis); and required intubation for daily respiratory support.

Clinical and bacteriologic evaluations: definitions of response

All patients receiving at least 1 dose of study drug were evaluated on an intent-to-treat basis. Antimicrobial effectiveness was evaluated on the bases of the clinical response of the patient and the bacteriologic response of the organism. Clinical and bacteriologic assessments were conducted during therapy (days 3-4), at the end of therapy, and at followup (7-10 days after treatment). Identification of causative organisms was performed using standard methods and susceptibility tests according to the Etest and standard procedures approved by the National Committee for Clinical Laboratory Standards (NCCLS).

To be considered evaluable for clinical response, patients must have satisfied the following requirements: 1) met all inclusion/exclusion criteria, 2) received at least 3 days of treatment with study drugs, and 3) completed an end-of-treatment or post-treatment assessment. The primary clinical response end point was success or failure at the end of initial therapy. Specific criteria for determining the clinical response of patients with pneumonia, UTI, sepsis, and intraabdominal infections are outlined in Table 1. In general, success with initial therapy was defined as resolution of all acute signs and symptoms and improvement, but no deterioration, in radiographic and laboratory abnormalities. Failure with initial therapy was defined as persistence or progression of signs and symptoms relevant to the original infection after 3 to 4 days of therapy; development of new clinical findings consistent with active infection; progression of radiographic and/ or laboratory abnormalities; or death due to the original infection. Clinical response was considered indeterminate under the following circumstances: modification of the initial therapy or administration of nonstudy antimicrobials before the 3- to 4-day ontreatment evaluation; early withdrawal from the study 
Table 1. Criteria for response to treatment in patients with infection

\begin{tabular}{|c|c|}
\hline Infection & Response criteria \\
\hline Pneumonia & $\begin{array}{l}\text { Improvement or normalization of respiratory status as measured } \\
\text { by respiratory rate, oxygenation, severity of cough, and sputum } \\
\text { production when compared with day } 1 \text {, and at least } 1 \text { of the following: } \\
\text { Normalization of body temperature } \\
\text { Reduction or resolution of left shift of WBCs on differential } \\
\text { Nonprogression or resolution of chest x-ray findings }\end{array}$ \\
\hline UTI & $\begin{array}{l}\text { Improvement or resolution of clinical signs and symptoms when } \\
\text { compared with day } 1 \text {, and at least } 1 \text { of the following: } \\
<10 \mathrm{WBCs} / \mathrm{HPF} \text { and }<10^{4} \mathrm{CFUs} / \mathrm{mL} \text { in microscopic } \\
\text { evaluation of spun urine } \\
\text { Reduction or resolution of left shift of WBCs on differential } \\
\text { Normalization of body temperature }\end{array}$ \\
\hline Sepsis & $\begin{array}{l}\text { Normalization or improvement of body temperature, heart rate, } \\
\text { and respiratory rate; improvement of clinical signs and symptoms } \\
\text { when compared with day } 1 \text {; and at least } 1 \text { of the following: } \\
\text { Reduction or resolution of left shift of WBCs on differential } \\
\text { Return of appetite }\end{array}$ \\
\hline $\begin{array}{l}\text { Intra-abdominal infections (such as } \\
\text { abdominal abscess and peritonitis) }\end{array}$ & $\begin{array}{l}\text { Normalization or improvement of body temperature and } \\
\text { improvement or resolution of clinical signs and symptoms, such as: } \\
\text { Abdominal pain/discomfort } \\
\text { Abdominal tenderness } \\
\text { Normalization or peristalsis and peristaltic sounds }\end{array}$ \\
\hline
\end{tabular}

CFU: colony-forming unit; HPF: high power field; WBC: white blood cell.

before the 3- to 4-day on-treatment evaluation; or inability to complete the study because of drug-related adverse events.

To be evaluated for bacteriologic response and to represent the clinical response population, the patient must have met the evaluation criteria and must have had a positive pretreatment culture for a bacterial pathogen obtained immediately prior to receipt of the first dose of study antibiotic, or during the first 48 hours following randomization. Bacteriologic response was determined to be eradication/presumed eradication (causative organism absent or no material to culture in a patient who was clinically cured), persistence/ presumed persistence (causative organism present or no material to culture in a patient whose clinical response was failure), or indeterminate (e.g. patient received a nonstudy systemic antibiotic with activity against the initial pathogen, or pathogen was resistant to the study medication).

Safety evaluation

The safety of study drug therapy was monitored by clinical observation and by conventional laboratory tests from the first antibiotic dose up to 30 days after treatment. Adverse events were rated by the investigator according to their severity (mild, moderate, severe) and their relationship to the study drug (certainly, probably, possibly, or not likely related, or unrelated). 
Table 2. Baseline demographics and medical characteristics of intent-to-treat population

\begin{tabular}{lcc}
\hline & $\begin{array}{c}\text { Cefepime } \\
(\mathbf{N}=\mathbf{1 5 9})\end{array}$ & $\begin{array}{c}\text { Comparator } \\
\mathbf{( N = 1 5 8 )}\end{array}$ \\
\hline Women, No. (\%) & $77(48)$ & $84(53)$ \\
Race, No. (\%) & $64(40)$ & $47(30)$ \\
$\quad$ White & $75(47)$ & $91(58)$ \\
Hispanic & $17(11)$ & $16(10)$ \\
Black & $3(2)$ & $4(3)$ \\
Other & $57(18-91)$ & $47(16-97)$ \\
Age, median (range) years & $18(11)$ & $21(13)$ \\
Received antibacterial pretherapy, No. (\%) & & $95(60)$ \\
Clinical diagnosis, No. (\%) & $101(64)$ & $35(22)$ \\
CAP & $30(19)$ & $18(11)$ \\
UTI & $20(13)$ & $10(6)$ \\
Intra-abdominal infection & $8(5)$ & $2(1)$ \\
Sepsis & & $116(73)$ \\
APACHE II scores*, No. (\%) & $39(25)$ \\
Mild (5) & $3(2)$ & \\
Moderate (5-12) & $115(72)$ & \\
Severe ( $\geq 13)$ & $37(23)$ & \\
\hline
\end{tabular}

*Missing responses excluded from calculations. APACHE II: Acute Physiology and Chronic Health Evaluation II; CAP: community-acquired pneumonia; UTI: urinary tract infection.

Table 3. Drug-related adverse events occurring in $\geq 2 \%$ of patients, $N(\%)$

\begin{tabular}{lcc}
\hline Adverse event & $\begin{array}{c}\text { Cefepime } \\
(\mathbf{N = 1 5 9 )}\end{array}$ & $\begin{array}{c}\text { Comparator } \\
(\mathbf{N = 1 5 8 )}\end{array}$ \\
\hline Any event & $25(16)$ & $30(19)$ \\
Phlebitis & $5(3)$ & $6(4)$ \\
Reaction at IV site & $3(2)$ & - \\
Fever & $2(1)$ & $4(3)$ \\
Diarrhea & $4(3)$ & $1(<1)$ \\
Nausea & $1(<1)$ & $4(3)$ \\
Abdominal pain & $3(2)$ & $4(3)$ \\
Rash & $1(<1)$ & $5(3)$ \\
Tachycardia & - & $6(4)$ \\
\hline
\end{tabular}


Figure 1. Treatment administration

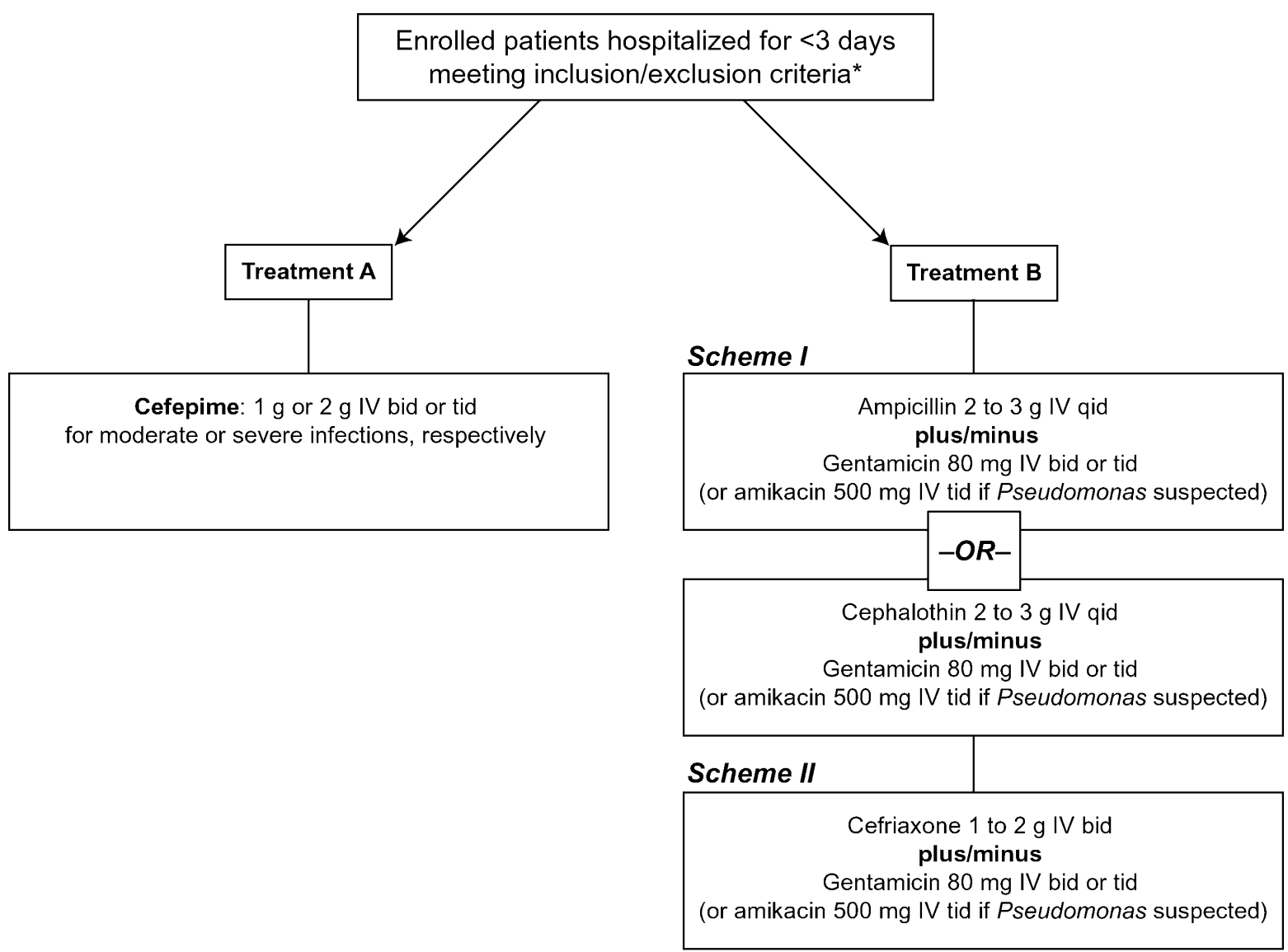

\section{Additional antibiotics that could be added to Treatments $A$ and $B$}

- Metronidazole IV if anaerobes suspected (intra-abdominal infection and aspiration pneumonia)

- Vancomycin IV if methicillin-resistant Staphylococcus aureus suspected or identified

- Macrolides IV if atypical pathogens suspected (patients with pneumonia)

*See text for inclusion/exclusion criteria.

Ethical issues

Each patient provided written informed consent following approval of the protocol by each institution's internal review board and in accordance with the Declaration of Helsinki.
Statistical analysis

The primary measure of efficacy was the overall proportion of patients in each treatment group with clinical success vs failure at the end of initial antibiotic therapy. Secondary efficacy analysis included both 
Figure 2. Clinical cure at end of treatment: clinically evaluable population

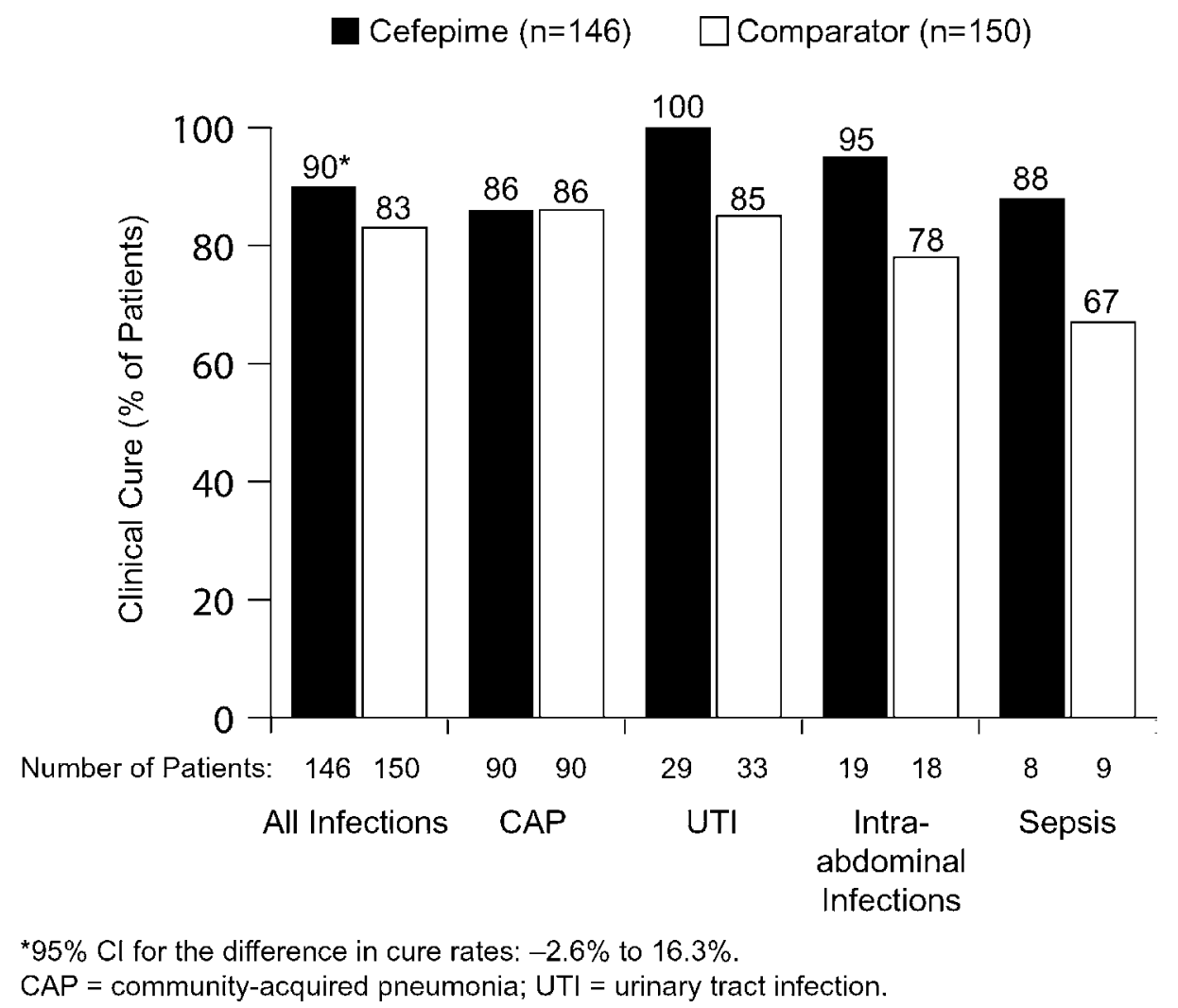

bacteriologic eradication rates for those with a pretreatment pathogen and clinical cure rates by infection type.

For the end-of-therapy clinical and bacteriologic responses, 95\% 2-sided confidence intervals (95\% CIs) were calculated for the mean differences between resolutions or eradication rates. Each of the 2 treatment comparisons was declared equivalent at the $2.5 \%$ significance level if the lower boundary was $\geq 15 \%$ CI. These analyses were performed for both the efficacy-evaluable and intent-to-treat populations.

For demographic and baseline medical characteristics, descriptive statistics were performed for the frequencies and percentages of categorical variables, numbers of patients, means, standard deviations, and minimum and maximum values for continuous variables. The incidence rates of adverse events and reasons for premature discontinuation were tabulated by both treatment group and body system.

\section{Results}

Three hundred twenty hospitalized patients with severe infections were enrolled at 34 clinical sites; 3 patients were not eligible for efficacy or safety analysis because no study drug was received. Therefore, 317 
patients constituted the intent-to-treat population (196 pneumonia, 65 UTI, 38 intra-abdominal infection, 18 sepsis). A total of 21 additional patients were excluded from evaluation of efficacy (13 cefepime, 8 comparator). Patients were ineligible for efficacy analysis on the basis of 1) pretreatment with antimicrobials (6 cefepime, 1 comparator) or 2) no test-of-cure evaluation (7 cefepime, 7 comparator). Accordingly, a total of 296 (93\%) patients were considered evaluable for the efficacy analysis (146 cefepime, 150 comparator). Five patients ( 3 cefepime, 2 comparator) were included in the efficacy population despite failure to meet entry criteria (i.e. baseline APACHE II scores $=5$ ). Four patients ( 2 with UTI and 2 with sepsis) had an APACHE II score equal to 5, and 1 patient with a UTI had an APACHE II score of 4.

Forty-three patients ( 26 cefepime, 17 comparator) were prematurely discontinued from the study. The most common reasons for premature discontinuation of study drug were death (13 cefepime, 6 comparator), an adverse event (4 cefepime, 4 comparator), patient noncompliance ( 3 cefepime, 3 comparator), treatment failure ( 3 cefepime, 3 comparator), protocol violation (2 cefepime, 1 comparator), and laboratory abnormality ( 1 cefepime).

Baseline demographics and medical characteristics of the intent-to-treat population are outlined in Table 2. The median age of the intent-to-treat group was 57 years. Overall, approximately $51 \%$ of patients were women and 52\% were Hispanic; more Hispanic patients were randomly assigned to comparator therapy (58\%) compared with cefepime therapy $(47 \%)$. A total of $12 \%$ of patients (11\% cefepime, $13 \%$ comparator) received previous antibacterial therapy. A majority of patients had a clinical diagnosis of CAP ( $64 \%$ cefepime, $60 \%$ comparator), followed by UTI ( $19 \%$ cefepime, $22 \%$ comparator), intraabdominal infection (13\% cefepime, $11 \%$ comparator), and sepsis (5\% cefepime, $6 \%$ comparator). Based on APACHE II scores, both groups had similar numbers of patients with moderate ( $72 \%$ cefepime, $73 \%$ comparator) and severe $(23 \%$ cefepime, $25 \%$ comparator) infection. The population of clinically evaluable patients had similar baseline demographics and medical characteristics compared with the intent-to-treat population; there were no differences across treatment groups for any infection site.

\section{Clinical efficacy}

The mean duration of antimicrobial therapy for both treatment groups was 10 days ( 9 cefepime, 10 comparator), with a range from 1 to 69 days. Compared with the comparator group, a greater number of patients from the cefepime group received treatment for $=10$ days $(66 \%$ cefepime, $55 \%$ comparator). Clinical responses stratified by treatment and country were very similar, with the exception of Mexico, where clinical cure was $90 \%$ for cefepimetreated patients compared with $78 \%$ for patients receiving a comparator regimen. Clinical cure rates for the intent-to-treat population were $82 \%$ for cefepime and $80 \%$ for the comparator group.

Clinical response at the end of treatment by infection type is shown in Figure 2 for the clinically evaluable population. Regardless of infection type, clinical cure rates were higher among cefepime-treated patients (90\%) compared with those given a comparator regimen $(83 \%)(95 \% \mathrm{CI}:-2.6 \%$ to $16.3 \%)$. For patients with CAP, both treatments provided an $86 \%$ clinical cure rate. Cefepime provided higher clinical cure rates compared with comparator regimens for patients diagnosed with UTI (100\% vs $85 \%)$, intra-abdominal infection (95\% vs 78\%), and sepsis ( $88 \%$ vs $67 \%$ ).

\section{Bacteriologic efficacy}

One hundred sixty-two pathogens (76 cefepime, 86 comparator) were isolated from clinically evaluable patients. The most common pathogens isolated were Escherichia coli $(\mathrm{n}=49)$, Streptococcus pneumoniae $(\mathrm{n}=29)$, Haemophilus influenzae $(\mathrm{n}=14)$, Klebsiella pneumoniae $(\mathrm{n}=6)$, and $S$. aureus $(\mathrm{n}=11)$, accounting for $64 \%$ of all isolated pathogens. None of the $E$. coli produced extended $\beta$-lactamases (ESBLs). In contrast, 2 isolates of $K$. pneumoniae were ESBL producers, 1 of which had intermediate susceptibility 
to cefepime, and the other full resistance to cefepime. All but 1 isolate of $S$. pneumoniae were fully susceptible to penicillin.

A total of $97 \%(74 / 76)$ of pathogens isolated from cefepime-treated patients were eradicated compared with $96 \%(81 / 84)$ in the comparator group. In the cefepime group, 2 pathogens persisted. One patient with CAP had presumed persistent $\beta$-lactamasenegative $H$. influenzae; this patient was a clinical failure. A second patient with UTI had presumed persistent $K$. pneumoniae and was also a clinical failure. Two patients treated with a comparator regimen had 3 persistent/presumed persistent pathogens at the end of treatment. One patient with CAP had presumed persistent $\beta$-lactamase-negative $H$. influenzae and Moraxella catarrhalis and was a clinical failure. The second patient had persistent $\beta$-lactamase $H$. influenzae but was considered clinically cured. Bacteriologic response was indeterminate for 2 pathogens in the comparator group.

\section{Safety and tolerance}

Three hundred seventeen patients (159 cefepime, 158 comparator) were eligible for the safety analysis. Eighty-four (53\%) cefepime- and 81 (51\%) comparator-treated patients reported at least 1 treatment-emergent event. Twenty-five (16\%) cefepime and 30 (19\%) comparator recipients had at least 1 adverse event considered to be drug-related (Table 3 ). For cefepime, injection site reactions, phlebitis, diarrhea, and abdominal pain were the most common drug-related adverse events reported. For the comparator regimens, phlebitis, fever, nausea, rash, and tachycardia represented the most frequently reported drug-related adverse events. Most adverse events were mild to moderate in severity ( 25 cefepime, 28 comparator) and improved or resolved without intervention.

Four cefepime- and 4 comparator-treated patients were prematurely discontinued owing to occurrence of 1 or more adverse events. Three events ( 1 cefepime, 2 comparator) were considered to be probably or definitely related to the study drug. The cefepimetreated patient was discontinued early owing to a urinary yeast infection. The 2 comparator-treated patients had premature discontinuation of therapy secondary to "loss of sensitivity" in the infusion arm or development of rash of moderate intensity.

A total of $27(9 \%)$ patients died during the course of the study (17 cefepime, 10 comparator). The majority of deaths ( $86 \%$ ) occurred in patients diagnosed with CAP before treatment began. Deaths were not considered treatment related, but were due to underlying disease and comorbidity.

\section{Discussion}

The main finding of this multicenter study was that empiric monotherapy with cefepime was at least as effective as conventional broad-spectrum antimicrobial combinations for treatment of moderate to severe community-acquired infections. For all infections combined, the clinical cure rate at the end of therapy was higher for those given cefepime alone (90\%) compared with those receiving comparator combination regimens $(83 \%)$. Furthermore, patients treated with cefepime who had a diagnosis of UTI, intra-abdominal infection, or sepsis had higher rates of clinical cure compared with comparator recipients. Approximately half of the clinically evaluable population had an isolated pathogen that was identified before treatment began. Cefepime eradicated $97 \%$ of all pathogens, similar to comparator therapy (94\%).Accordingly, this study extends the findings of previously published clinical studies, which found cefepime to be effective for the treatment of moderate to serious infections of the lower respiratory tract [19-23] and the urinary tract [24], as well as intraabdominal infections [25] and bacteremia [26].

This study also demonstrated that cefepime has an excellent safety and tolerability profile with adverse events primarily limited to injection site reactions (e.g. phlebitis) and effects on the gastrointestinal tract (e.g. diarrhea, abdominal pain). Importantly, most cefepimerelated adverse events were of mild $(76 \%)$ or moderate (24\%) intensity. Only 1 cefepime-treated patient, a 38 year-old black female, discontinued the drug prematurely owing to "loss of sensitivity" in the arm receiving the drug infusion. 
CAP was the most frequent diagnosis in this study, accounting for approximately two thirds of the 317 moderate to severe infections. The cefepime and comparator regimens were similarly effective, demonstrating an $86 \%$ clinical cure rate at the end of therapy. The most up-to-date guidelines published by the Infectious Diseases Society of America and the American Thoracic Society recommend a thirdgeneration cephalosporin (e.g. ceftriaxone) plus a macrolide as 1 option for treatment of hospitalized patients with CAP $[27,28]$. Among nearly 1,800 bacterial isolates obtained from patients with community-acquired respiratory tract infections in several Latin American countries, the average rate of penicillin-resistant S. pneumoniae was 39\% [9]. Notably, the highest susceptibility rates were found in Argentina (77\%) and Brazil (72\%), and the lowest rate of penicillin susceptibility was detected in Mexico $(33 \%)$. In the same surveillance study, rates of $\beta$ lactamase-positive $H$. influenzae and $M$. catarrhalis were approximately $13 \%$ and $92 \%$, respectively. The administration of an advanced-generation cephalosporin with the addition of a macrolide as empiric therapy covers the most likely causes of lower respiratory tract infection, including $S$. pneumoniae, $H$. influenzae, and atypical pathogens. In clinical practice, many physicians omit the macrolide if infection with an atypical pathogen is deemed unlikely. Accordingly, cefepime monotherapy is a reasonable choice for hospitalized patients with CAP of moderate to severe intensity who are not considered to be at risk for infections with atypical respiratory pathogens.

In a second surveillance study conducted in Latin America, less than half of $E$. coli isolates obtained from hospitalized patients with UTIs were susceptible to broad-spectrum penicillins; in vitro resistance rates were also high against both old and new fluoroquinolones [10]. Cefepime monotherapy was also found to be clinically and bacteriologically effective in the management of hospitalized patients with moderate to severe UTIs. Clinical cure was 100\% following cefepime vs $85 \%$ for the comparator regimen. Arecent surveillance study, conducted in Latin America among hospitalized patients with UTI, found that cefepime had excellent in vitro susceptibility against many antibiotic-resistant urinary $E$. coli isolates (91.7\%). Because antimicrobial resistance among uropathogens causing community-acquired UTIs (e.g. E. coli) is increasing [29], especially against ampicillin and trimethoprim-sulfamethoxazole (TMP-SMX), new antibiotic options are needed to treat these infections $[30,31]$. The data from our trial are promising; it appears that cefepime monotherapy is an effective empiric agent for the treatment of potentially antibioticresistant uropathogens and serious UTIs.

Treatment of intra-abdominal infection requires early diagnosis, timely and properly performed surgical procedures, and appropriately selected antibacterial agents to reduce the incidence of peritonitis, abscess, or local wound infection [32]. In this multicenter study, 37 clinically evaluable patients had intra-abdominal infections. Notably, cefepime-treated patients had a higher clinical cure rate (95\%) compared with those who received combination antibiotic therapy $(78 \%)$. Because of the small number of patients enrolled and evaluable in this trial, firm conclusions cannot be reached regarding the efficacy of cefepime (plus agents with anaerobic coverage) for treatment of serious intraabdominal infections. However, our findings appear to confirm an earlier report by Barie, et al. in which cefepime plus metronidazole was associated with high cure rates $(88 \%)$ in patients with severe intra-abdominal infections [25].

Patients with sepsis must be diagnosed early and treated promptly if death is to be prevented [33]. The antimicrobial regimen empirically selected typically has been of broad spectrum and includes activity against E. coli, K. pneumoniae, and P. aeruginosa. Although combination $\beta$-lactam/aminoglycoside regimens have been given frequently, new monotherapy options with excellent pseudomonal coverage are now more often prescribed, especially when renal toxicity is a concern $[3,7]$. A third Latin American surveillance study found that bloodstream infection isolates (e.g. E. coli) were uniformly more resistant to all classes of tested antimicrobial agents compared with similar isolates recovered from North American patients [11]. In this study, only 17 patients with sepsis ( 8 cefepime, 9 
comparator) were evaluable for clinical efficacy. At the end of therapy, clinical cure was $88 \%$ for cefepime compared with $67 \%$ for comparator regimens. Despite the inadequate numbers of evaluable patients, cefepime's excellent in vitro activity against the organisms commonly associated with sepsis makes it a reasonable empiric treatment option.

Few clinical studies describe the "real-world" approach to treatment of serious infections with empiric antimicrobial therapy. Limitations of this study included the nonblinded design, the large number of centers, the variety of comparator agents studied, and the low number of patients in several of the infection subgroups. Although these limitations necessitate careful interpretation, evaluation of clinical success at the end of therapy reflects "real-world" clinical practice and is noteworthy.

In summary, the relatively recent availability of potent broad-spectrum antimicrobial agents has decreased the need for combination antimicrobial therapy for the treatment of hospitalized patients with serious infections. This study enrolled "real-world" hospitalized patients with moderate to severe community-acquired infections, who were treated with either cefepime monotherapy or a $\beta$-lactam \pm aminoglycoside therapy. Empiric use of cefepime monotherapy resulted in high rates of clinical success and bacteriologic eradication and was at least as effective as standard combination regimens for treatment of patients with CAP. For the subgroups of evaluable patients with UTI, intra-abdominal infection, and sepsis, cefepime monotherapy appeared to provide higher clinical cure rates compared with the comparator combination antimicrobial regimens. Overall, cefepime given alone was effective and well tolerated in the treatment of hospitalized adult patients with moderate to severe community-acquired infections.

If they are to prescribe appropriate empiric therapy, physicians must be knowledgeable about geographic differences in the causes and susceptibility patterns of organisms associated with serious community-acquired infections. Use of inappropriate empiric antimicrobial therapy may lead to increased rates of mortality and morbidity. As with any serious infection, antimicrobial therapy should be tailored when culture and susceptibility data become available.

\section{Acknowledgement}

We are indebted to Dr. Eduardo Rodenas for his strong support in the organization and construction of the LAARG. Also, we would like to thank the members of the Medical Division of Bristol Myers Squibb Brasil for their excellent coordination of the trial.

\section{List of investigators}

Daniel Stanboulian, Clínica Sagrada Familia Sanatorio Otamendi, Buenos Aires, Argentina; Dario Quinodos and Ernesto Jakob, Hospital Italiano de Córdoba, Córdoba, Argentina; Maria José Furst, Sanatorio Méndez, Buenos Aires, Argentina; Roberto Badaró, Hospital Universitário Professor Edgard Santos, Universidade Federal da Bahia, Salvador, Bahia, Brazil; João Silva de Mendonça, Hospital do Servidor Público Estadual, São Paulo, Brazil; Helio Sader, Universidade Federal de São Paulo-EPM, São Paulo, Brazil; Valdir Golin, Santa Casa de Misericórdia de São Paulo, São Paulo, Brazil; Willie Oigman, Hospital Pedro Ernesto (UERJ), Rio de Janeiro, Brazil; Antonio Tarcisio Freire, Santa Casa de Misericórdia de Belo Horizonte, Belo Horizonte, Brazil; Carlos Starling, Hospital São Francisco de Assis, Belo Horizonte, Brazil; Alexandre Tsanaclis, Hospital Beneficência Portuguesa, São Paulo, Brazil; Alexandre Zilenovski, Hospital do Campo Limpo, São Paulo, Brazil; Otavio Messerder, Hospital Português, Salvador, Brazil; João Carlos Correa, Hospital da Venerável Ordem Terceira de São Francisco da Penitência, Rio de Janeiro, Brazil; Guido Levi, Instituto de Infectologia Emílio Ribas, São Paulo, Brazil; Maria Cassia Correa, Hospital das Clínicas de São Paulo, São Paulo, Brazil; Lausane Mayrinck, Hospital da Venerável Ordem Terceira de São Francisco da Penitência, Rio de Janeiro, Brazil; Marcia Noya Rabelo, Hospital São Rafael, Salvador, Brazil; Mauro Sánchez Mena, Hospital de 
Especialidades Centro Médico Nacional, I.M.S.S., Mérida, Yuc., México; Alejandro Macias, Hospital General Regional de León, Gto., León, Gto., México; Luis Guillermo Juárez Martínez, Hospital General de Zona \#33 I.M.S.S., Monterrey, N.L., México; Francisco Márquez, Instituto de Salud del Estado de Aguascalientes "Hospital Miguel Hidalgo," Aguascalientes, Ags., México; Fernando Molinar Ramos, Hospital de Especialidades, C.M. "La Raza," I.M.S.S., México, D.F.; Isidro Zavala, Hospital Universitario “Dr. Angel Leaño," Universidad Autónoma de Guadalajara, Guadalajara, Jal., México; Carlos Dillman, Hospital San José, TEC de Monterrey, Monterrey, N.L., México; Juan Carlos Tinoco, Hospital General de Durango, S.S., Durango, Dgo., México; Martin Mendoza Rodríguez, Hospital Pediátrico Villa, S.S., México, D.F., México; Carlos Seas, Hospital Cayetano Heredia-Instituto de Medicina Tropical, Lima, Peru.

\section{References}

1. Moellering R.C. Principles of anti-infective therapy. In: Mandell G.L., Bennett J.E., Dolin R. eds. Principles and practice of infectious diseases, 5th ed. New York: Churchill-Livingstone, 2000.

2. Fernandez-Guerrero M., Gudiol F., Rodriguez-Torres A., et al. Nosocomial pneumonia: comparative multicentre trial between monotherapy with cefotaxime and treatment with antibiotic combinations. Infection 1991;19(suppl 6):S320-5.

3. Mounton Y.J., Buscart C. Empirical monotherapy with meropenem in serious bacterial infections. Meropenem Study Group. J Antimicrob Chemother 1995;36(suppl A):145-56.

4. Costa S.F., Newbaer M., Santos C.R., et al. Nosocomial pneumonia: importance of recognition of aetiological agents to define an appropriate initial empirical therapy. Int J Antimicrob Agents 2001; 17:147-50.

5. Dranitsaris G., Tran T.M., McGeer A., Narine L. Pharmacoeconomic analysis of empirical therapy with ceftazidime alone or in combination antibiotics for febrile neutropenia in cancer patients. Pharmacoeconomics 1995; 7:49-62.

6. Plosker G.L., Foster R.H., Benfield P. Cefotaxime. A pharmacoeconomic review of its use in the treatment of infections. Pharmacoeconomics 1998;13(1 Pt 1):91-106.
7. Young M., Plasker G.L. Piperacillin/tazobactam: a pharmacoeconomic review of its use in moderate to severe bacterial infections. Pharmacoeconomics 2001;19:1135-75.

8. Hoban D.J., Doern G.V., Fluit A.C., et al. Worldwide prevalence of antimicrobial resistance in Streptococcus pneumoniae, Haemophilus influenzae, and Moraxella catarrhalis in the SENTRY Antimicrobial Surveillance Program, 1997-1999. Clin Infect Dis 2001;32(suppl 2):S81-93.

9. Sader H.S., Gales A.C., Granacher T.D., et al. Prevalence of antimicrobial resistance among respiratory tract isolates in Latin America: results from SENTRY antimicrobial surveillance program (1997-98). Braz J Infect Dis 2000; 4:245-54.

10. Gales A.C., Jones R.N., Gordon K.A., et al. Activity and spectrum of 22 antimicrobial agents tested against urinary tract infection pathogens in hospitalized patients in Latin America: report from the second year of the SENTRY antimicrobial surveillance program (1998). J Antimicrob Chemother 2000;45:295-303.

11. Diekema D.J., Pfaller M.A., Jones R.N., et al. Survey of bloodstream infections due to gram-negative bacilli: frequency of occurrence and antimicrobial susceptibility of isolates collected in the United States, Canada, and Latin America for the SENTRY Antimicrobial Surveillance Program, 1997. Clin Infect Dis 1999;29:595-607.

12. Wynd M.A., Paladino J.A. Cefepime: a fourth-generation parenteral cephalosporin. Ann Pharmacother 1996;30:1414-24.

13. Segreti J., Levin S. Bacteriologic and clinical applications of a new extended-spectrum parenteral cephalosporin. Am J Med 1996;100(6A):45S-51S.

14. Cunha B.A., Gill M.V. Cefepime. Med Clin North Am 1995;79:721-32.

15. Hardin T.C., Jennings T.S. Cefepime. Pharmacotherapy 1994; 14:657-68.

16. Barradell L.B., Bryson H.M. Cefepime. A review of its antibacterial activity, pharmacokinetic properties and therapeutic use. Drugs 1994;47:471-505.

17. Sanders C.C. Cefepime: the next generation? Clin Infect Dis 1993; 17:369-79.

18. Knaus W.A., Draper E.A., Wagner D.P., Zimmerman J.E. APACHE II: a severity of disease classification system. Crit Care Med 1985; 13:818-29.

19. Lin J.C., Yeh K.M., Peng M.Y., Chang F.Y. Efficacy of cefepime versus ceftazidime in the treatment of adult pneumonia. J Microbiol Immunol Infect 2001;34:131-7.

20. Bonfitto P., Lamorgese V., De Vietro T., et al. A randomized trial of cefepime and ceftazidime for the treatment of community-acquired pneumonia. J Chemother 1999;11:273-7. 
21. Grossman R.F., Campbell D.A., Landis S.J., et al. Treatment of community-acquired pneumonia in the elderly: the role of cefepime, a fourth-generation cephalosporin. J Antimicrob Chemother 1999; 43:549-54.

22. Borja J., Jane F. Cefepime versus ceftriaxone for empiric treatment of hospitalized patients with communityacquired pneumonia. Antimicrob Agents Chemother 1999;43:436-7.

23. Zervos M., Nelson M. Cefepime versus ceftriaxone for empiric treatment of hospitalized patients with community-acquired pneumonia. The Cefepime Study Group. Antimicrob Agents Chemother 1998;42:729-33.

24. Gentry L.O., Rodriguez-Gomez G. Randomized comparison of cefepime and ceftazidime for treatment of skin, surgical wound, and complicated urinary tract infections in hospitalized subjects. Antimicrob Agents Chemother 1991;35:2371-4.

25. Barie P.S., Vogel S.B., Dellinger E.P., et al. A randomized, double-blind clinical trial comparing cefepime plus metronidazole with imipenem-cilastatin in the treatment of complicated intra-abdominal infections. Cefepime Intra-abdominal Infection Study Group. Arch Surg 1997; 132:1294-302.

26. Schrank J.H. Jr., Kelly J.W., McAllister C.K. Randomized comparison of cefepime and ceftazidime for treatment of hospitalized patients with gram-negative bacteremia. Clin Infect Dis 1995;20:56-8.

27. Barlett J.G., Dowell S.F., Mandell L.A., et al. Practice guidelines for the management of community-acquired pneumonia in adults. Clin Infect Dis 2000;31:347-82.

28. Niederman M.S., Mandell L.A., Anzueto A., et al. Guidelines for the management of adults with community-acquired pneumonia. Diagnosis, assessment of severity, antimicrobial therapy, and prevention. Am J Respir Crit Care Med 2001; 163:1730-54.

29. Gupta K., Scholes D., Stamm W.E. Increasing prevalence of antimicrobial resistance among uropathogens causing acute uncomplicated cystitis in women. JAMA 1999;281:736-8.

30. Warren J.W., Abrutyn E., Hebel J.R., et al. Guidelines for antimicrobial therapy of uncomplicated acute bacterial cystitis and acute pyelonephritis in women. Clin Infect Dis 1999;29:745-58.

31. Gupta K., Hooton T.M., Stamm W.E. Increasing antimicrobial resistance and the management of uncomplicated community-acquired urinary tract infections. Ann Intern Med 2001;135:41-50.

32. McClean K., Sheehan G., Harding G. Intra-abdominal infection: a review. Clin Infect Dis 1994;9:100-6.

33. Periti P. Current treatment of sepsis and endotoxaemia. Expert Opin Pharmacother 2000;1:1203-17. 